inger, New

ability and . $1-10$.

st-neighbor

sess. Stoch.

istics. $A d v$.

jints. Proc.

ts. Preprint

in two and

Assoc. 77,

\title{
A REMARK ON THE VAN LIESHOUT AND BADDELEY $J$-FUNCTION FOR POINT PROCESSES
}

\author{
T. BEDFORD,* Delft University of Technology \\ J. VAN DEN BERG, ${ }^{* *} C W I$
}

\begin{abstract}
The empty space function of a stationary point process in $\mathbb{R}^{d}$ is the function that assigns to each $r, r>0$, the probability that there is no point within distance $r$ of $O$. In a recent paper Van Lieshout and Baddeley study the so-called $J$-function, which is defined as the ratio of the empty space function of a stationary point process and that of its corresponding reduced Palm process. They advocate the use of the $J$-function as a characterization of the type of spatial interaction.

Therefore it is natural to ask whether $J \equiv 1$ implies that the point process is Poisson. We restrict our analysis to the one-dimensional case and show that a classical construction by Szász provides an immediate counterexample. In this example the interpoint distances are still exponentially distributed. This raises the question whether it is possible to have $J \equiv 1$ but non-exponentially distributed interpoint distances. We construct a point process with $J \equiv 1$ but where the interpoint distances are bounded.
\end{abstract}

POINT PROCESS; J-FUNCTION; EMPTY SPACE FUNCTION; POISSON PROCESS

AMS 1991 SUBJECT CLASSIFICATION: PRIMARY 60G55; 60J05; 60G10

\section{Introduction and statement of the main result}

We will start by giving a rather informal introduction to some of the notions we need. For a formal treatment see, for instance, Daley and Vere-Jones (1988).

Let $\mu$ be the distribution of a stationary point process. The corresponding Palm process is obtained by 'conditioning on having a point in the origin'. (By a 'point' we will always mean a point of the point process.) It is well-known that if $\cdots, \tau_{-1}$, $\tau_{0}=0, \tau_{1}, \cdots$ denote the points of the Palm process, then the sequence of interpoint distances $\left(X_{i}, i \in \mathbb{Z}\right)$, defined by $X_{i}=\tau_{i}-\tau_{i-1}, i \in \mathbb{Z}$ is stationary. (One can also reverse the above procedure, so that each stationary sequence of non-negative random variables with finite expectations gives rise to a stationary point process.) Further, the reduced Palm process is simply obtained from the Palm process by removing the point at the origin. The distribution of the reduced Palm process will be denoted by $\mu$ !

Received 29 January 1996; revision received 7 June 1996.

* Postal address: Delft University of Technology, Faculty of Technical Mathematics and Informatics, Mekelweg 4, 2628 CD Delft, The Netherlands.

** Postal address: CWI, Kruislaan 413, 1098 SJ Amsterdam, The Netherlands. 
In a recent paper Van Lieshout and Baddeley (1996) introduced the function $J$ defined by

$$
J(r)=\frac{\mu^{\prime}(\text { no point in } B(O, r))}{\mu(\text { no point in } B(O, r))}, \quad 0<r<\infty,
$$

where $B(O, r)$ is the closed ball with center $O$ and radius $r$. It is important to note that for a Poisson point process $\mu=\mu^{\prime}$, and hence $J \equiv 1$. To express the dependence of $J$ on $\mu$ we will sometimes write $J_{\mu}$.

The $J$-function was presented as a useful tool in spatial statistics (see also Stoyan et al. (1995), p. 121). In particular, Van Lieshout and Baddeley advocate its use as a (non-parametric) measure of spatial interaction. However, our results indicate that some care has to be taken in the use of this function as it is unable to distinguish between two very different types of point processes.

Van Lieshout and Baddeley write that $J<1$ suggests a 'clustered' pattern, $J>1$ suggests a 'regular' pattern, while $J \equiv 1$ can be interpreted as lack of interaction. Because of the last it is a natural question whether $J \equiv 1$ implies that the process is Poisson.

In this paper we restrict to the one-dimensional case. We first show that a result of Szász (1970) immediately implies that the answer to the question above is negative. Szász proves that for every $k$ there exists a point process (whose distribution we will denote by $\mu_{k}$ ) on $\mathbb{R}$, which is not Poisson, but has the property that, for all pairwise disjoint finite intervals $U_{1}, U_{2}, \cdots, U_{k}$, the joint distribution of the number of points in $U_{i}, 1 \leqslant i \leqslant k$, is the same as for the Poisson point process with density 1 . Note that we have, for a stationary point process on $\mathbb{R}$ with distribution $\mu$,

(2) $J_{\mu}(r)=\frac{\mu^{!}(\text {no point in }[-r, r])}{\mu(\text { no point in }[-r, r])}$

$$
\begin{aligned}
& =\lim _{\varepsilon \rightarrow 0} \frac{\mu(\text { no point }[-r, \varepsilon] \text { and no point in }[\varepsilon, r] \mid \text { one point in }(-\varepsilon, \varepsilon))}{\mu(\text { no point in }[-r, r])} \\
& =\lim _{\varepsilon \rightarrow 0} \frac{\mu \text { (no point in }[-r, r] \text {, one point in }(-\varepsilon, \varepsilon) \text {, no point in }[\varepsilon, r])}{\mu \text { (one point in }(-\varepsilon, \varepsilon)) \mu(\text { no point in }[-r, r])}
\end{aligned}
$$

However, for $\mu=\mu_{3}$, for every $\varepsilon>0$ each probability in the last expression is the same as for the Poisson point process with density 1 . Hence

$$
J_{\mu_{3}} \equiv J_{\text {Poisson }}=1 \text {. }
$$

Inherent in this example is the fact that the interpoint distances are exponentially distributed. Therefore, the next natural question is whether there exists a point process with $J \equiv 1$ but whose interpoint distances are not exponentially distributed. We show that this is indeed the case. In our example the interpoint distances are even bounded. 
:e

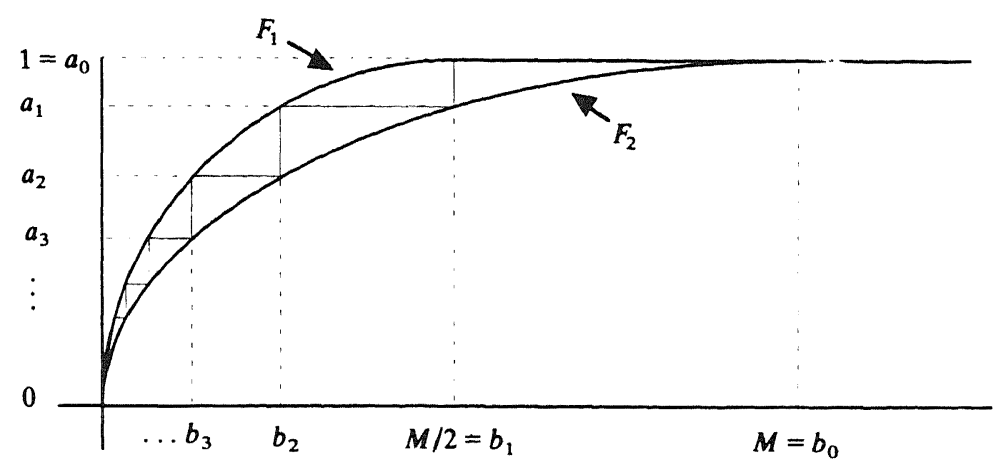

Figure 1

Theorem 1. There exist suitable $M>0, c>0$ and an ergodic, stationary, symmetric point process which has $J \equiv 1$, and for which the interpoint distances have expectation 1 and probability density $c /(1+x)^{2}$ on $[0, M]$ and 0 outside this interval.

Remarks. (i) Random variables with density proportional to $1 /(1+x)^{2}$ on a bounded interval are called shifted truncated Pareto random variables (with shape parameter 1). (ii) It will appear that $M \approx 3.92$ and $c \approx 1.26$.

\section{Proof of Theorem 1}

Consider a stationary point process with distribution $\mu$, and let, as before, $X_{i}$, $i \in \mathbb{Z}$ be the stationary sequence of interpoint distances for the associated Palm process. It is clear that if $J \equiv 1$ then, after spatial scaling, we still have $J \equiv 1$. Therefore we will assume that the $X_{i}$ have expectation 1 . We will investigate what $J \equiv 1$ means in terms of the interpoint distances. Clearly (using the notation of Section 1),

$$
\begin{aligned}
\mu^{\prime}(\text { no point in }[-r, r]) & =\boldsymbol{P}\left(\tau_{-1}<-r, \tau_{1}>r\right) \\
& =\boldsymbol{P}\left(X_{0}>r, X_{1}>r\right)=\boldsymbol{P}\left(X_{1}>r, X_{2}>r\right) .
\end{aligned}
$$

Now, for a Borel set $B$, let $N(B)$ denote the number of points in $B$ for the stationary point process, and let $N_{0}(B)$ be the analog for the Palm process. We have, of course,

$$
\mu(\text { no point in }[-r, r])=\boldsymbol{E}[I(N([-r, r])=0)],
$$

where $I(\cdot)$ denotes the indicator function. From a standard result relating a stationary point process to its Palm process (see, e.g., equation (12.3.7) of Daley and Vere-Jones (1988)) we get immediately that the right-hand side in (6) equals

$$
\boldsymbol{E}\left[\int_{0}^{X_{1}} I\left(N_{0}([x-r, x+r])=0\right) d x\right] .
$$

However, the above integral is evidently equal to $\left(X_{1}-2 r\right)^{+}$, where we use the 
notation $a^{+}$for $\max (a, 0)$. So we have $\mu$ (no point in $\left.[-r, r]\right)=\boldsymbol{E}\left(X_{1}-2 r\right)^{+}$, and get the following lemma.

Lemma 1. Let $\cdots, X_{-1}, X_{0}, X_{1}, \cdots$ be a stationary sequence of non-negative random variables with $E\left(X_{0}\right)=1$. Then the stationary point process whose Palm process has the above sequence $\left(X_{i}, i \in \mathbb{Z}\right)$ as interpoint distances, has $J \equiv 1$ if and only if

$$
\forall r>0, \quad \boldsymbol{P}\left(X_{1}>r, X_{2}>r\right)=E\left(X_{1}-2 r\right)^{+} .
$$

Remark. It is easy to check that, for the Poisson process with intensity $1,(7)$ is indeed satisfied.

Our strategy now is to construct a pair of random variables $\left(X_{1}, X_{2}\right)$ whose distribution is symmetric and satisfies (7) in Lemma 1 , and whose marginal distributions are as in Theorem 1. Moreover, our construction must have the property that the conditional distribution of $X_{2}$, given $X_{1}$, is sufficiently 'spread-out' so that we can extend the pair $\left(X_{1}, X_{2}\right)$ to a Markov chain $\cdots X_{-1}, X_{0}, X_{1}, \cdots$, that is stationary and ergodic. By Lemma 1 this sequence gives rise to a point process as mentioned in Theorem 1.

Our construction uses the following lemma, which in turn is inspired by the construction used in Theorem 1 of Bedford and Meilijson (1993).

Lemma 2. Let $M>0$, and let $F_{1}$ and $F_{2}:[0, \infty] \rightarrow[0,1]$ be continuous functions satisfying $F_{1}(0)=F_{2}(0)=0, F_{1} \equiv 1$ on $[M / 2, x) F_{2} \equiv 1$ on $[M, x), F_{1}$ strictly increasing and differentiable on $(0, M / 2), F_{2}$ strictly increasing and differentiable on $(0, M)$, and $F_{1}>F_{2}$ on $(0, M)$.

Then there exists a pair of non-negative random variables $\left(X_{1}, X_{2}\right)$ such that

(a) $\left(X_{1}, X_{2}\right)$ has a symmetric distribution;

(b) $\boldsymbol{P}\left(X_{1}=X_{2}\right)=0$;

(c) $\boldsymbol{P}\left(X_{1}<t, X_{1}<X_{2}\right)=\frac{1}{2} F_{1}(t), t \in[0, x)$;

(d) $\boldsymbol{P}\left(X_{1}<t, X_{1}>X_{2}\right)=\frac{1}{2} F_{2}(t), t \in[0, \infty)$;

(e) $\left(X_{1}, X_{2}\right)$ can be extended to a stationary ergodic sequence $\cdots, X_{-1}, X_{0}, X_{1}, X_{2}, \cdots$.

Remark. In fact, the sequence $X_{i}, i \in \mathbb{Z}$, in our construction will be a Markov chain.

Proof of Lemma 2. See Figure 1. Let $a_{0}=1$ and $a_{i}=F_{2} F_{1}^{-1}\left(a_{i-1}\right), i \geqq 1$ (where $\left.F_{1}^{-1}(1):=M / 2\right)$. Then $a_{n} \downarrow 0$ as $n \uparrow x$. To construct $\left(X_{1}, X_{2}\right)$, first draw a non-negative integer $i$ with probability $a_{i}-a_{i+1}$. Then draw $U_{1}$ and $U_{2}$ uniformly and independently on the interval $\left(a_{i+1}, a_{i}\right)$. Finally, with probability $1 / 2$ take $X_{1}=F_{1}^{-1}\left(U_{1}\right)$ and $X_{2}=F_{2}^{-1}\left(U_{2}\right)$ (call this choice 1 ) and with probability $1 / 2$ take $X_{1}=F_{2}^{-1}\left(U_{2}\right)$ and $X_{2}=F_{1}^{-1}\left(U_{1}\right)$ (choice 2).

We now prove the properties (a)-(e). 
(a) This follows immediately from the (last step in the) above construction.

(b) Note that given the value of $i$ in the above construction, the random variable $F_{2}^{-1}\left(U_{2}\right)$ is (conditionally) independent of $F_{1}^{-1}\left(U_{1}\right)$ and has a continuous distribution (because $F_{2}$ is strictly increasing on $(0, M)$ ). This implies the result. (Alternatively: $F_{1}^{-1}\left(a_{i+1}, a_{i}\right)$ and $F_{2}^{-1}\left(a_{i+1}, a_{i}\right)$ are disjoint. However this is no longer true after the adaptation in the remark below.)

(c) First note that, in the construction above, $U_{1}$ and $U_{2}$ are uniformly distributed on $(0,1)$. Now

$$
\left(X_{1}<X_{2}, X_{1}<t\right) \Leftrightarrow\left(X_{1}=F_{1}^{-1}\left(U_{1}\right), X_{1}<t\right) \Leftrightarrow\left(U_{1}<F_{1}(t) \text { and choice } 1\right),
$$

which has probability $\frac{1}{2} F_{1}(t)$.

(d) This is analogous to (c).

(e) Let $\left(X_{1}, X_{2}\right)$ be as in the construction above. Given $X_{2}=x$, choose $X_{3}$ randomly with distribution $P\left(X_{2} \in \cdot \mid X_{1}=x\right)$. (This conditional probability is properly defined because of the differentiability condition in the statement of this lemma.) Then, given $X_{3}=y$, choose $X_{4}$ randomly, with distribution $P\left(X_{2} \in . \mid X_{1}=\right.$ $y$ ) etc. Analogously, construct $X_{0}, X_{-1}$ etc. This is clearly a stationary sequence (in fact, it is a reversible stationary Markov chain). To show that this sequence is ergodic, we have to show that there is 'sufficient communication' between different parts of the state space $[0, M]$. To see this, define $b_{i}=F_{2}^{-1}\left(a_{i}\right), i \geqq 0$. Note that, for $i \geqq 1$, this is also equal to $F_{1}^{-1}\left(a_{i-1}\right)$. We have $b_{0}=M>b_{1}=M / 2>b_{2}>b_{3}>\cdots$, and $b_{n} \rightarrow 0$ as $n \rightarrow \infty$. This yields a partition of $[0, M]$ in intervals $\left(b_{1}, b_{0}\right]$, $\left(b_{2}, b_{1}\right], \cdots$.

Now let $i \geqq 1$ and $x \in\left(b_{i+1}, b_{i}\right]$. Given $X_{1}=x$, there is a positive probability that choice 1 was made in the above construction, i.e. $X_{2}=F_{2}^{-1}\left(U_{2}\right)$ with $U_{2}$ uniformly distributed in $\left(a_{i}, a_{i-1}\right]$. Hence $X_{2}$ has (conditional) positive density on $\left(b_{i}, b_{i-1}\right]$. But there is also a positive probability that choice 2 was made, which, in an analogous way, shows that $X_{2}$ also has positive (conditional) density on $\left(b_{i+2}, b_{i+1}\right]$. In the same way we see that, given $X_{1}=x \in\left(b_{1}, b_{0}\right], X_{2}$ has positive density on $\left(b_{2}, b_{1}\right]$. We conclude that for each $i$ and $j \geqq 0$ and each $x \in\left(b_{i+1}, b_{i}\right]$, there exists a $k$ such that, given $X_{1}=x, X_{k+1}$ has positive density on $\left(b_{j+1}, b_{j}\right]$ (namely, if $i \neq j$ take $k=|i-j|$, else take $k=2$ ). This guarantees the 'amount of communication' mentioned above and hence the required ergodicity.

This completes the proof of Lemma 2.

Remark. Note that, in the above construction, we have a certain periodicity: given $X_{1}=x \in\left(b_{i+1}, b_{i}\right], X_{k}$ has positive density on $\left(b_{i+1}, b_{i}\right]$ for even $k$, but 0 density for odd $k$. This does not disturb our argument, since we only need stationarity and ergodicity in the ergodic-theoretical sense (i.e. not in the Markov chain theoretical sense which includes aperiodicity). However, with a small adaptation of our construction we can also get aperiodicity: instead of taking each $a_{i+1}$ equal to $F_{2} F_{1}^{-1}\left(a_{i}\right)$, we can take the sequence $\left(a_{i}, i \in N\right)$ so that $F_{1}^{-1}\left(a_{i-1}\right)<F_{2}^{-1}\left(a_{i}\right), i \geqq 1$, and 
$F_{2}^{-1}\left(a_{i}\right)<F_{1}^{-1}\left(a_{i-2}\right), i \geqq 2$. In the previous construction $F_{1}^{-1}\left(\left(a_{i+1}, a_{i}\right)\right)$ was exactly $F_{2}^{-1}\left(\left(a_{i+2}, a_{i+1}\right)\right)$ which 'caused' the above mentioned periodicity. But in the new construction $F_{1}^{-1}\left(\left(a_{i+1}, a_{i}\right)\right)$ intersects $F_{2}^{-1}\left(\left(a_{i+2}, a_{i+1}\right)\right)$ and $F_{2}^{-1}\left(\left(a_{i+3}, a_{i+2}\right)\right)$, which provides a 'more flexible communication' and aperiodicity.

Proof of Theorem 1. It is clear that for every $M>0$ there exists a unique $c>0$ such that

$$
\int_{0}^{M} \frac{c}{(1+x)^{2}} d x=1
$$

i.e. $c /(1+x)^{2}$ is the probability density of a random variable with support $M$. Moreover, a few lines of standard calculations show that there is a unique pair $(M, c)$ such that this random variable has expectation 1, i.e.

$$
\int_{0}^{M} \frac{c x}{(1+x)^{2}} d x=1
$$

(In fact, $c$ is the solution of $c\left(1-\exp ^{-2 / c}\right)=1$, which appears to be $\approx 1.26$, and $M=1 /(c-1) \approx 3.92$; or, equivalently, $M$ satisfies $\log (1+M)=2 M /(1+M)$.) From now on we work with this choice of $c$ and $M$.

Let $Y$ be a random variable with the above probability density. In particular, $E Y=1$. Define, for $x \geq 0$,

$$
F_{1}(x)=1-\boldsymbol{E}(Y-2 x)^{+},
$$

and

$$
F_{2}(x)=1+\boldsymbol{E}(Y-2 x)^{+}-2 \boldsymbol{P}(Y>x) .
$$

Claim. $F_{1}$ and $F_{2}$ satisfy the conditions of Lemma 2.

Proof of claim. The conditions that involve only $F_{1}$ follow directly from the above definitions. The facts that $F_{2}$ is differentiable, $F_{2}(0)=0$ and $F_{2}(M)=1$ also follow immediately. Further, $F_{2}$ is clearly strictly increasing on $[M / 2, M]$. Differentiation (with respect to $x$ ) shows that the condition that $F_{2}$ is also strictly increasing on $[0, M / 2]$ is equivalent to

$$
\frac{1}{1+M}>\frac{1}{1+2 x}-\frac{1}{(1+x)^{2}}, \quad 0<x<M / 2,
$$

which can be checked by differentiating once more. Finally, the condition $F_{1}(x)>$ $F_{2}(x), x \in(0, M)$, is clearly true for $x \geqq M / 2$ (because $F_{1} \equiv 1$ on $[M / 2, M]$, $F_{2}(M)=1$, and $F_{2}$ is strictly increasing on $\left.[M / 2, M]\right)$. For $x \leqq M / 2$ it is equivalent to (evaluate the integrals in the definition of $F_{1}$ and $F_{2}$ )

$$
\frac{1}{1+x}+\log (1+2 x)-\frac{2 x}{1+M}>\log (1+M)-\frac{M-1}{M+1}, \quad 0<x \leqq M / 2,
$$

which can be checked by standard arguments: differentiating several times with 
respect to $x$ shows that the minimum of the left-hand side of $(10)$ in the interval $[0, M / 2]$ can only be taken at $x=0$ (where we already know directly from the definitions that $F_{1}=F_{2}$ and hence that the left-hand side of (10) equals its right-hand side) or at $x=M / 2$, for which we can easily check that (10) holds. This completes the proof of the above claim.

Now application of Lemma 2 yields a stationary ergodic sequence $\left(X_{i}, i \in \mathbb{Z}\right)$ with

$$
\begin{aligned}
\boldsymbol{P}\left(X_{1}<t\right) & =\boldsymbol{P}\left(X_{1}<t, X_{1}<X_{2}\right)+\boldsymbol{P}\left(X_{1}<t, X_{1}>X_{2}\right) \\
& =\frac{1}{2}\left(F_{1}(t)+F_{2}(t)\right) \\
& =\boldsymbol{P}(Y<t) .
\end{aligned}
$$

The first equality follows from part (b) of Lemma 2, the second from part (c) and (d) of Lemma 2, and the last equality from (8) and (9). So each $X_{i}$ has probability density $c /(1+x)^{2}$ on $(0, M)$ and 0 outside.

We also have

$$
\begin{aligned}
\boldsymbol{P}\left(X_{1}>t, X_{2}>t\right) & =2 \boldsymbol{P}\left(X_{2}>X_{1}>t\right) \\
& =2\left(\boldsymbol{P}\left(X_{1}<X_{2}\right)-\boldsymbol{P}\left(X_{1}<X_{2}, X_{1}<t\right)\right) \\
& =1-F_{1}(t) \\
& =\boldsymbol{E}\left(X_{1}-2 t\right)^{+} .
\end{aligned}
$$

Here the first equation follows from part (a) of Lemma 2, the third from part (c), and the last from (8) and (11).

Now application of Lemma 1 completes the proof of Theorem 1.

\section{Acknowledgements}

We thank (in chronological order) M. Keane for raising the questions which led to this research, and for some useful discussions, M. Van Lieshout for referring us to an article by P. Moran (which, in turn, referred to the article by Szász mentioned above), P. Spreij for bringing us together, and O. J. Boxma for comments on the first version of this paper.

\section{References}

BEDFORD, T. AND MEILIJSON, I. (1993) A characterisation of marginal distributions of (possibly dependent) lifetime variables which right censor each other. Report 93-116. TU Delft.

Daley, D. J. AND Vere-Jones, D. (1988) An Introduction to the Theory of Point Processes. Springer, New York.

VAN LIESHOUT, M. N. M. AND BADDELEY, A. (1996) A nonparametric measure of spatial interaction in point patterns. Statist. Neerlandica. 50, 344-361.

Stoyan, D., Kendall, W. S. And Mecke, J. (1995) Stochastic Geometry and its Applications. 2nd edn. Wiley, New York.

SzÁsz, D. (1970) Once more on the Poisson process. Studia Sci. Math. Hungarica 5, 441-444. 